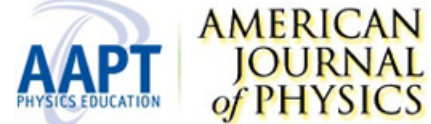

\section{Laser interferometric characterization of a vibrating speaker system}

A. A. Freschi, N. R. Caetano, G. A. Santarine, and R. Hessel

Citation: American Journal of Physics 71, 1121 (2003); doi: 10.1119/1.1586262

View online: http://dx.doi.org/10.1119/1.1586262

View Table of Contents: http://scitation.aip.org/content/aapt/journal/ajp/71/11 ?ver=pdfcov

Published by the American Association of Physics Teachers

\section{AAPT Re-register for Table of Content Alerts}

\section{Create a profile. Sign up today!}




\title{
Laser interferometric characterization of a vibrating speaker system
}

\author{
A. A. Freschi, ,a) N. R. Caetano, G. A. Santarine, and R. Hessel \\ Departamento de Física, IGCE, UNESP, Caixa Postal 178, 13500-970, Rio Claro, SP, Brazil
}

(Received 4 November 2002; accepted 2 May 2003)

\begin{abstract}
An experiment that combines opto-mechanical and electrical measurements for the characterization of a loudspeaker is presented. We describe a very simple laser vibrometer for evaluating the amplitude of the vibration (displacement) of the speaker cone. The setup is essentially a Michelson-type interferometer operated by an inexpensive semiconductor laser (diode laser). It is shown that the simultaneous measurements of three amplitudes (displacement, electrical current, and applied voltage), as functions of the frequency of vibration, allow us to characterize the speaker system. The experiment is easy to perform, and it demonstrates several useful concepts of optics, mechanics, and electricity, allowing students to gain an intuitive physical insight into the relations between mathematical models and an actual speaker system. (C) 2003 American Association of Physics Teachers.
\end{abstract}

[DOI: $10.1119 / 1.1586262]$

\section{INTRODUCTION}

Since the advent of the laser in the early 1960s it has been natural to study vibrations by means of the Doppler effect. ${ }^{1}$ The idea is to use an optical interferometer to measure the frequency shift of a coherent laser beam that is scattered from a small area of the vibrating object. The interferometer mixes the scattered light with a reference beam, resulting in an optical signal whose (beat) frequency is equal to that of the difference between the mixed beams. By using this procedure both the velocity and/or the displacement of the object can be precisely measured. Several variations of the laser Doppler technique have been developed for applications in fields such as structural dynamic testing, acoustics, quality control, industrial plants, and medical diagnosis. ${ }^{1-4}$ As a consequence of the wide acceptance of the technique, the related instruments (usually called Laser Doppler Vibrometers) have become important tools for those involved either in research or applications involving experimental vibration and dynamic system analysis. However, despite the fact that there is extensive coverage on this subject in the technical literature, there are relatively few papers devoted to the use of the laser Doppler technique in undergraduate laboratories. ${ }^{5-8}$

This work presents a very simple laser vibrometer suitable for characterizing electromechanic transducers such as dynamic loudspeakers. The aim is to give the student a general introduction to the Doppler effect, optical interference, and vibration analysis, by illustrating an application of the laser Doppler technique in the field of acoustics. Vibration measurements on a speaker system are one of the typical cases where, because of the speaker cone lightness, optical techniques are much preferred over the usual instrumentation (such as accelerometers).

We consider one of the most common speaker models, which consists of a light coil suspended on a strong permanent magnetic field fixed on the center of the moving cone. In Sec. II A the basic theoretical expressions relating electrical and mechanical quantities of the vibration of the coil in the magnetic field are derived. Section II B presents a general explanation of the Doppler effect and optical interferometry. It is assumed that the student has a thorough knowledge of the fundamental laws of mechanics and electromagnetism, and is familiar with calculus and complex numbers. Emphasis has been given to explaining the basic concepts together with a description of the experimental setup and components (in Sec. III). The results are presented and discussed in Sec. IV.

\section{THEORY}

\section{A. The dynamic loudspeaker}

A loudspeaker is a device that converts electrical energy into sound. ${ }^{9}$ The most common models consist of a moving cone firmly cemented to a small light cylindrical coil in its center, as depicted in Fig. 1. The coil is in a strong magnetic field of a permanent magnet. The lines of the radial magnetic field lie in a plane perpendicular to the $x$ axis shown in Fig. 1. Alternating current through the coil causes the speaker cone to vibrate, thus producing sound waves to the air.

Consider a loudspeaker in which the coil is fed with a steady sinusoidal voltage, $V$, of amplitude $V_{0}$ and angular frequency $\omega$. The alternating electric current $i$ flowing through the coil has amplitude $i_{0}$. The force $F$ on the coil (and the resulting motion) is in the $x$ axis direction and can be written as $F=B l i$, with $B$ the magnetic field strength at the coil, and $l$ the length of wire in the coil. Because the coil moves perpendicularly to the magnetic field, and the wire is perpendicular to the field and to the motion, there is an induced electromotive force given by $E=B l u$, where $u$ is the coil speed. ${ }^{9,10}$

If the loudspeaker vibrates with small amplitude (low input power), we may think of it as a linear system. ${ }^{10}$ Thus all oscillatory quantities can be expressed by sinusoidal functions with temporal angular frequency $\omega$. Let us call $x$ the position of the central point of the speaker cone along the $x$ axis, and define $x=0$ as its equilibrium position (which occurs in the absence of any external applied voltage). The amplitude of the displacement is $x_{0}$. As will be shown, it is possible to characterize the speaker system by fitting the experimental data of the current normalized amplitude of vibration, $x_{0} / i_{0}$, and the impedance, $Z_{0}=V_{0} / i_{0}$, as functions of $\omega$, using the corresponding theoretical expressions. A classical approach to obtain these expressions makes use of complex numbers to represent the physical parameters that oscillate. We write a little caret $\left({ }^{\wedge}\right)$ over the parameter to 


\section{Permanent magnetic field, $B$}

Coil: wire length, $l$ resistance, $R$ inductance, $L$

Mass (cone + coil), $m$

Overall elastic constant, $k$

Dissipation constant (friction + sound), $b$

Fig. 1. Speaker parameters and physical cross section

represent that it is a complex number; the corresponding physical quantity being the real part of the expression. Thus we may write,

$$
\hat{F}=B l \hat{i}
$$

and

$$
\hat{E}=B l \hat{u}=j \omega B l \hat{x}
$$

where the temporal derivative $d \hat{x} / d t$ was replaced by $j \omega \hat{x}(d / d t \rightarrow j \omega),{ }^{10}$ with $j=\sqrt{-1}$. Because the product $B l$ is real, the $j$ in Eq. (2) simply means that $E$ and $u$ lead the displacement $x$ by $90^{\circ}$.

We now write two more equations for our analysis. Let $R$ and $L$ be the electrical resistance and the inductance of the coil, respectively. The coil and the speaker cone altogether have mass $m$, the overall suspension elastic constant is $k$, and the dissipation constant (due to friction plus useful sound) is $b$. We assume that the speaker cone and the coil move together as a "rigid piston," which is a good approximation for low frequencies, at which the sound wavelength is long compared with the diameter of the speaker cone.,11,12 For the electrical part of the speaker, the applied voltage $V$ must overcome the induced electromotive force, the voltage across the resistor and the inductor,

$$
\hat{V}=\hat{E}+R \hat{i}+j \omega L \hat{i},
$$

with the voltage across the inductor $(L d i / d t)$ written as $j \omega L \hat{i}$. On the mechanical side, the force accelerating the mass $m$ can be expressed as

$$
\hat{F}-k \hat{x}-j \omega b \hat{x}=-m \omega^{2} \hat{x},
$$

where $-k x \rightarrow-k \hat{x}$ is the elastic force, $-b u=-b d x / d t$ $\rightarrow-j \omega b \hat{x}$ is the frictional plus air resistance force, and $m d^{2} x / d t^{2} \rightarrow-m \omega^{2} \hat{x}$ is the mass times acceleration. Note that the problem expressed by Eq. (4) represents a damped oscillator subjected to an external force $\hat{F}$, with $b$ the damping constant (which depends on the characteristics of both the speaker and the surrounding air). If we eliminate $\hat{F}$ in Eqs. (1) and (4) and calculate $\hat{x} / \hat{i}$, we obtain

$$
\frac{x_{0}}{i_{0}}=\left|\frac{\hat{x}}{\hat{i}}\right|=\frac{B l / k}{\left\{\left[1-\omega^{2} \frac{m}{k}\right]^{2}+\omega^{2} \frac{b^{2}}{k^{2}}\right\}^{1 / 2}}
$$

with the parameters $a_{1}=B l / k, a_{2}=m / k$, and $a_{3}=b / k$.

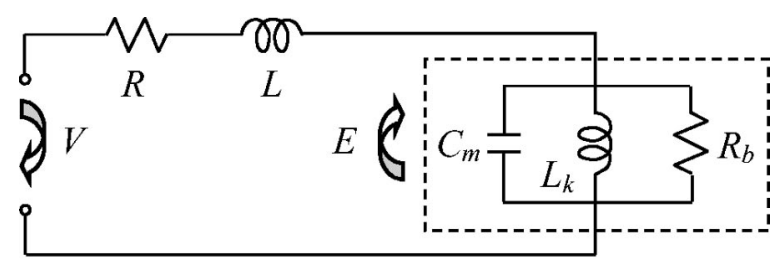

Fig. 2. Equivalent all-electrical network to a loudspeaker. The resistance of the wire is $R$ and $L$ is the inductance. The impedance inside the dashed box is called "motional impedance," with $C_{m}=m /(B l)^{2}, L_{k}=(B l)^{2} / k$, and $R_{b}$ $=(B l)^{2} / b$. The applied voltage is $V$ and $E$ is the induced electromotive force (in volts).

An important conclusion that may be drawn from Eq. (5) is the existence of a resonance at $\omega=[(\mathrm{k} / \mathrm{m})$ $\left.-\left(b^{2} / 2 m^{2}\right)\right]^{1 / 2}$. In practice, the damping constant is generally small $(b \ll \sqrt{\mathrm{km}})$, so the resonance occurs at $\omega$ $\approx \sqrt{k / m}$. Also, as we might expect, a strong magnetic field $B$, a long length of the wire in the coil $l$, and a small elastic coefficient $k$, result in greater motion, and the term $B l / k$ appears naturally in the numerator of Eq. (5).

The impedance $\hat{Z}=\hat{V} / \hat{i}$ can be calculated by solving simultaneously Eqs. (1)-(4). We eliminate $\hat{F}, \hat{E}$, and $\hat{x}$ :

$$
\begin{aligned}
Z_{0}=|\hat{Z}| & =\frac{V_{0}}{i_{0}}=\left\{\left[R+\frac{(B l)^{2} / b}{1+\frac{1}{\omega^{2}} \frac{k^{2}}{b^{2}}\left(1-\omega^{2} \frac{m}{k}\right)^{2}}\right]^{2}\right. \\
& \left.+\omega^{2}\left[L+\frac{(B l)^{2} / k}{\left(1-\omega^{2} \frac{m}{k}\right)+\frac{1}{\frac{1}{\omega^{2}} \frac{k^{2}}{b^{2}}\left(1-\omega^{2} \frac{m}{k}\right)}}\right]^{2}\right\}^{1 / 2}
\end{aligned}
$$

with three more independent parameters: $a_{4}=R, a_{5}=L$, and $a_{6}=(B l)^{2} / b$ [the last parameter, $(B l)^{2} / k$, can be calculated from the previous ones]. It is clear that the determination of the parameters $a_{1}$ to $a_{6}$ represents a solution for the speaker problem because the product $B l=a_{3} a_{6} / a_{1}$, and therefore the parameters $b, k$, and $m$, can be easily calculated.

In the analysis of loudspeakers it often is convenient to replace the actual speaker system by an equivalent motional electrical system. ${ }^{9}$ By writing $\hat{Z}=R+j \omega L+\hat{Z}_{M}$, it is possible to show that a parallel combination of a resistor, capacitor, and inductor can represent the term $\hat{Z}_{M}$, sometimes also called motional impedance. The equivalent electrical network is depicted in Fig. 2, where $C_{m}=m /(B l)^{2}$ is the analog of $m, L_{k}=(B l)^{2} / k$ is the analog of $k$, and $R_{b}=(B l)^{2} / b$ the analog of $b$. (This circuit is but one of a number of circuits that can be devised from the rationalization of the complex impedance function $\hat{Z}$.) From inspection of Fig. 2 we can tell that the frequency of resonance is defined primarily by $C_{m}$ and $L_{k}$ (indeed, $m / k=L_{k} C_{m}$ ). The term $b / k=L_{k} / R_{b}$ which appears in Eqs. (5), and (6) has dimension of time, and is related to the sharpness of the resonance peak. Of the total resistive component $R+R_{b}$ in this analogy, only that part given by $R_{b}$ is associated with the transfer of electrical en- 
ergy to acoustic energy (plus losses due to friction in flexing the speaker cone), whereas the energies stored in $L_{k}$ and $C_{m}$ correspond, respectively, to the mechanical potential and kinetic energy of the system. The average power $W$ dissipated as sound (plus frictional losses) is then given by $W$ $=E_{\mathrm{rms}}^{2} / R_{b}=b u_{\mathrm{rms}}^{2}$, with $E_{\mathrm{rms}}$ and $u_{\mathrm{rms}}$ the rms (root mean square) amplitudes of the voltage $E$ and the velocity $u$, respectively. Note that at $\omega=\sqrt{1 / L_{k} C_{m}}$, the same current flows through $R$ and $R_{b}$ (the currents through $L_{k}$ and $C_{m}$ cancel each other), and the ratio of the radiated sound power (plus frictional losses) to the total power dissipated by the speaker system is simply $R_{b} /\left(R+R_{b}\right)$. As we might expect from the analysis of the electrical network depicted in Fig. 2, the power dissipated by $R_{b}$ is greatly attenuated at low frequencies due to the shunting effect of $L_{k}$, and at high frequencies due to the shunting effect of $C_{m}$ (and also because the input current is blocked by $L$ ).

\section{B. Doppler effect and wave mixing}

The Doppler effect ${ }^{1}$ can be used to measure the velocity (and/or the displacement) of an object that scatters light. The idea is to detect the change in the frequency of an electromagnetic wave due to the relative motion of the object and the receiver. Consider a light beam sent out from a laser to an object moving with velocity $u$ in the direction of the beam. To first order in $u / c$, with $c$ the speed of light, the fractional shift in frequency of the backreflected beam is ${ }^{1} 2 u / c$. Thus, for an object moving at $1 \mathrm{~mm} / \mathrm{s}$, the frequency shift is 1 part in $1.5 \times 10^{11}$. Although extremely small, this shift (usually called the Doppler shift) can be precisely measured by mixing the reflected light from the object with a reference beam from the same laser.

A common problem in the laser Doppler technique is the discrimination of the direction of the velocity. This problem is usually solved by employing optical devices (such as Bragg cells) to shift the frequency of the reference beam. ${ }^{1}$ However, when only the amplitude of a sinusoidal vibration is of interest, all we need is an ordinary Michelson-type interferometer, ${ }^{13}$ which is the basis of the setup used in our experiments (Fig. 3). In this configuration, a laser beam is divided into a reference beam and a signal beam by the cube beamsplitter BS. The reference beam is directed onto a stationary object, $\mathrm{O}_{1}$, and the signal beam is directed onto the vibrating test object (loudspeaker), $\mathrm{O}_{2}$. The retro-reflected beams return to the beamsplitter, where part of the beam coming from $\mathrm{O}_{1}$ is transmitted, and part of the beam coming from $\mathrm{O}_{2}$ is reflected toward the photodetector $\mathrm{D}$. When the test object moves, the frequency of the signal beam is shifted, resulting in an optical power modulation of the mixed wave due to interference between the reference and signal beams.

The expression for the output voltage $V_{D}$ from the photodetector can be derived from the well-established wave analysis of two-beam interference ${ }^{6,13}$ and may be written as

$$
V_{D}=\kappa P_{0}\left\{1+m \cos \left[\frac{4 \pi}{\lambda}\left(x_{1}-x_{2}\right)\right]\right\},
$$

with $\kappa$ the voltage responsivity of the photodetector, $P_{0}$ the average optical power, $m$ the modulation depth, $\lambda$ the light wavelength, and $x_{1}$ and $x_{2}$ the optical path lengths of the two arms of the interferometer. Note that the path difference be-

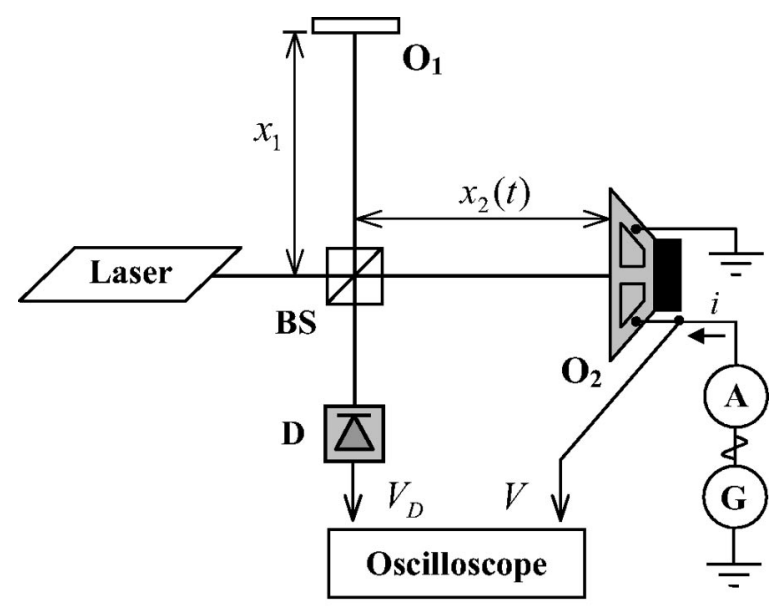

Fig. 3. Experimental setup, illustrating the interferometer and electrical instruments. BS: beam splitter, D: photodetector, $\mathrm{O}_{1}$ : static reference plate, $\mathrm{O}_{2}$ : loudspeaker, A: milliammeter, G: function generator. The applied voltage to the speaker is $V$, the current is $i$, the detector output is $V_{D}$, and $x_{1,2}$ are the arm lengths of the interferometer.

tween the signal and reference beams when they recombine is $2 x_{1}-2 x_{2}$, and anything that changes this path difference will cause a change in the output voltage $V_{D}$. Each complete cycle ( $2 \pi$ phase shift) on the alternating component of $V_{D}$ corresponds to an object movement of $\lambda / 2$ and the frequency of this modulation is the Doppler shift, given by $\nu_{D}$ $=2 u / \lambda$. Thus, by counting the number of complete cycles through which the movement of the object causes the output voltage to change, we can determine the total distance traveled by the object.

If the test object oscillates harmonically with amplitude $x_{0}$ and angular frequency $\omega$, we may let $x_{2}=x_{20}+x_{0} \sin (\omega t)$, so the total phase excursion in one period of vibration $T$ $=2 \pi / \omega$ is $16 \pi x_{0} / \lambda$. Therefore, if $x_{0} \gg \lambda$ and $n$ is the number of complete cycles described by the voltage $V$ in a time interval $T$, we may write $2 \pi n=16 \pi x_{0} / \lambda$, or

$$
x_{0}=n \lambda / 8 \quad\left(x_{0} \gg \lambda\right) .
$$

\section{EXPERIMENTAL REALIZATION}

We used a $3.5 \mathrm{~mW}$ optical output power semiconductor laser diode ${ }^{14}$ operating at $\lambda=0.65 \mu \mathrm{m}$ for the light source of the setup illustrated in Fig. 3. The diode laser module incorporates a mounted laser diode, adjustable focusing lens, and driver circuit into a compact cylindrical package $(12 \mathrm{~V}$ dc operating voltage). The cube beamsplitter ${ }^{15}$ transmits and reflects approximately $50 \%$ of the incoming light and the interferometer arms were set for equal lengths to within a few millimeters $\left(x_{1} \approx x_{2} \approx 15 \mathrm{~cm}\right)$. The laser coherence length ${ }^{13}$ was about $10 \mathrm{~cm}$, much larger than the beam's path imbalance. The laser beam is focused onto the reference $\left(\mathrm{O}_{1}\right)$ and test $\left(\mathrm{O}_{2}\right)$ objects: a small metallic plate and a low-power $(1$ $\mathrm{W})$ loudspeaker, respectively. The loudspeaker has nominally $8 \Omega$ impedance, with a speaker cone $60 \mathrm{~mm}$ in diameter. In order to measure the vibration of the coil, the laser beam is made normally incident (in the $x$-axis direction) in the center of the speaker cone. The photodetector (D) consists of an inexpensive silicon photodiode connected to a transimpedance amplifier integrated on a single monolithic chip. ${ }^{16}$ It 


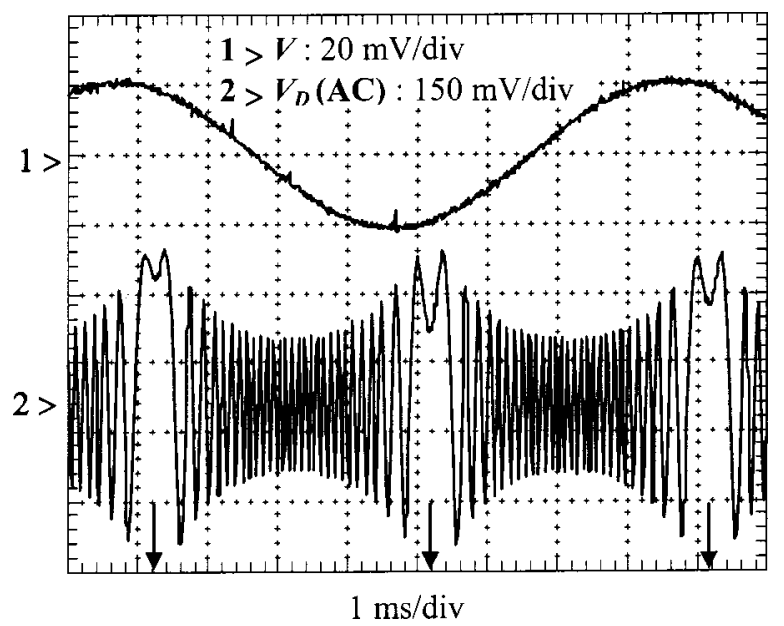

Fig. 4. Typical figure observed on the oscilloscope screen. Curve 1 shows the applied voltage $V$, and curve 2 shows the ac component of the detector output voltage, $V_{D}(\mathrm{ac})$. Each complete cycle (or peak) of the $V_{D}(\mathrm{ac})$ curve corresponds to a speaker cone displacement of $\lambda / 2$, or $0.325 \mu \mathrm{m}$. The amplitude of the vibration is obtained by counting the number of peaks in one (or half) period of vibration. The arrows on the time axis show the locations that correspond to instantaneous zero velocity, $u=0$ (and the position $x$ $\left.= \pm x_{0}\right)$.

was enclosed in a small aluminum box, which has a small hole for collecting the incoming light beam. The output voltage, $V_{D}$, is the product of the photodiode current and an external feedback resistor, $R_{F}$, and is proportional to the radiant power falling on the photodiode's active area $(A$ $\left.=5.2 \mathrm{~mm}^{2}\right)$. At the wavelength of $0.65 \mu \mathrm{m}$ and $R_{F}$ $=10 \mathrm{M} \Omega$, the full photodetector voltage responsivity is approximately $\kappa=4.5 \mathrm{~V} / \mu \mathrm{W}$.

All components, with the exception of the loudspeaker, were mounted on a small aluminum breadboard. The breadboard was placed on a foam sheet to reduce the noise inserted by mechanical vibrations in the optical setup. A droplet of reflective ink ${ }^{17}$ was placed on the illuminated areas of both objects (reference plate and loudspeaker) to increase the collected light power, and the detected voltage accordingly. The loudspeaker is fed with a steady sinusoidal voltage of amplitude $V_{0}$ and frequency $\nu=\omega / 2 \pi$ from a function generator $(\mathrm{G}){ }^{18}$ An ammeter ${ }^{19}$ (A) measures the current $i_{\text {rms }}$ $=i_{0} / \sqrt{2}$ through the speaker (see Fig. 3). The input impedance of the oscilloscope is set to $1 \mathrm{M} \Omega$, much higher than the impedance of the loudspeaker. The voltage $V$ that feeds the loudspeaker, and the ac component of the output voltage from the photodetector $\left[V_{D}(\mathrm{ac})\right]$, are visualized on an oscilloscope $^{20}$ screen (triggered using $V$ as reference).

\section{RESULTS AND DISCUSSION}

A typical measurement is illustrated in Fig. 4, where little more than one vibrating period was acquired in the oscilloscope. The experimental input is the sinusoidal applied voltage $V$ (curve 1 in Fig. 4). In the example, the frequency $\nu=125 \mathrm{~Hz}$ and the amplitude $V_{0}=20 \mathrm{mV}$. The measured current $i_{0}=2.36 \mathrm{~mA}$. The amplitude $x_{0}$ is determined by counting the number of peaks (complete cycles) in a halfperiod of the $V_{D}$ (ac) signal (curve 2 in Fig. 4). This number can be underestimated by a maximum of 1 peak, so high precision demands a large number of peaks to be counted.
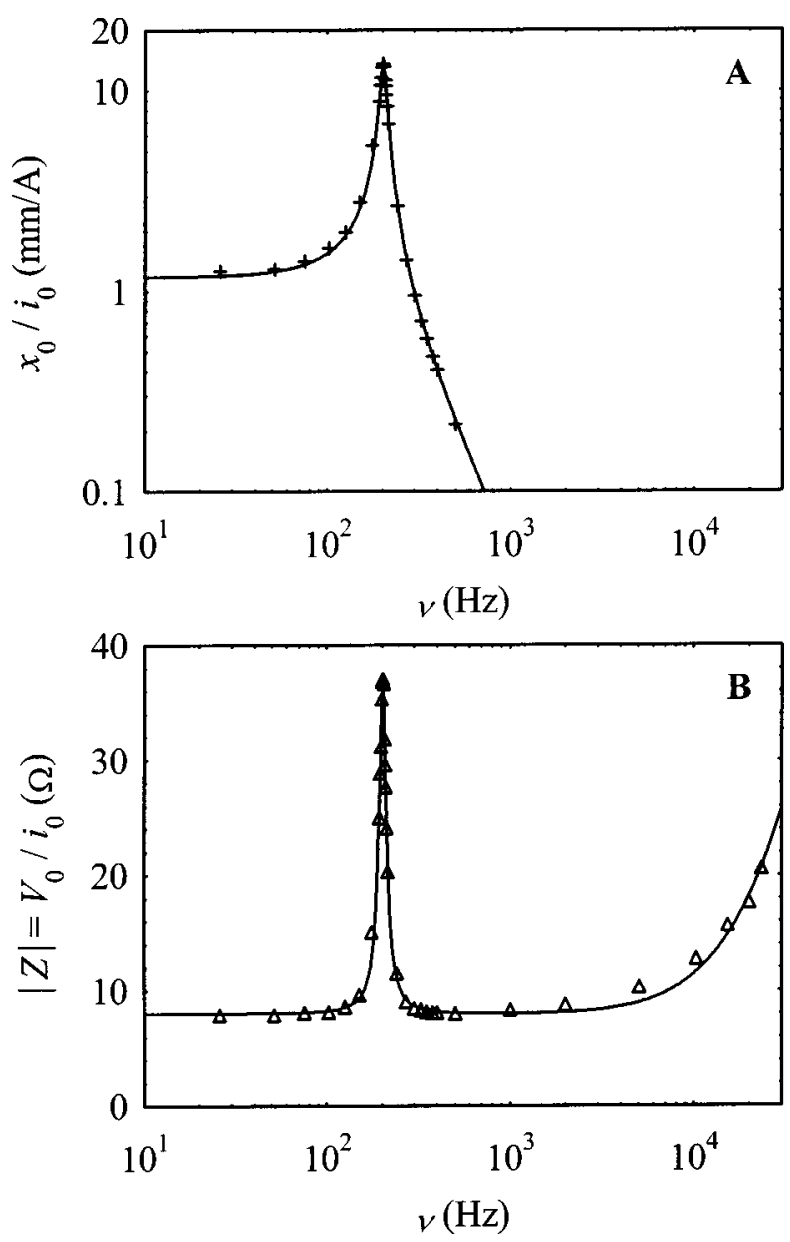

Fig. 5. Experimental results (dots) and fitted curves (solid lines). (a) Current normalized amplitude of vibration, $x_{0} / i_{0}$, and (b) amplitude of the impedance, $Z_{0}=V_{0} / i_{0}$, as functions of the vibrating frequency.

All our measurements involved more than 25 peaks in a half-period $(n / 2>25)$, so $x_{0}$ was always calculated with a precision better than $4 \%$. Three arrows mark on the horizontal axis of Fig. 4 (time axis: 1 ms/division) the locations that correspond to instantaneous zero velocity, $u=0$ (and the position $\left.x= \pm x_{0}\right)$. Note that the amplitude of the $V_{D}$ signal drops as the Doppler shift (and the velocity $u$ ) increases due to the finite frequency bandwidth of the photodetector. It is not a problem as long as the maximum Doppler shift is not far beyond bandwidth. If desirable, larger bandwidths may be achieved at the expense of lower responsivity by reducing the resistance of the feedback resistor $R_{F}$ of the photodetector. In Fig. 4, the number of complete cycles (or peaks) in a half-period of vibration is $n / 2=28$, which results in $x_{0}$ $=n \lambda / 8=4.6 \mu \mathrm{m}$. Thus at $\nu=125 \mathrm{~Hz}, x_{0} / i_{0}=1.93 \mathrm{~mm} / \mathrm{A}$, and $V_{0} / i_{0}=8.47 \Omega$.

The current normalized amplitude of vibration $x_{0} / i_{0}$ was measured for frequencies ranging from 25 to $500 \mathrm{~Hz}$, and the impedance $Z_{0}=V_{0} / i_{0}$ was measured up to $30 \mathrm{kHz}$. The results are illustrated in Fig. 5. The dots represent the experimental data whereas the solid curves correspond to the fittings with Eq. (5) in Fig. 5(a), and Eq. (6) in Fig. 5(b). The fittings were performed using a least-squares routine and the uncertainties were estimated by calculating the variances. ${ }^{21}$ From the first fitting [Fig. 5(a)] we get $a_{1}=B l / k=(1.15$ $\pm 0.04) \mathrm{mm} / \mathrm{A}, \quad a_{2}=m / k=(6.23 \pm 0.02) \times 10^{-7} \mathrm{~s}^{2}$, and $a_{3}$ 
Table I. Computed values of the speaker system (MKS units). Definitions of the parameters are given in the text and are summarized in Figs. 1 and 2.

\begin{tabular}{|c|c|c|c|c|}
\hline $\begin{array}{c}B l \\
(\mathrm{~T} \times \mathrm{m})\end{array}$ & $\begin{array}{c}b \\
(\mathrm{~N} \times \mathrm{s} / \mathrm{m})\end{array}$ & \multicolumn{2}{|c|}{$\begin{array}{c}m \\
\left(\times 10^{-3} \mathrm{~kg}\right)\end{array}$} & $\begin{array}{c}k \\
\left(\times 10^{3} \mathrm{~N} / \mathrm{m}\right)\end{array}$ \\
\hline $1.73 \pm 0.16$ & $0.10 \pm 0.02$ & 0.94 & .13 & $1.50 \pm 0.19$ \\
\hline $\begin{array}{c}R \\
(\Omega)\end{array}$ & $\begin{array}{c}L \\
\left(\times 10^{-4} \mathrm{H}\right)\end{array}$ & $\begin{array}{c}C_{m} \\
\left(\times 10^{-6} \mathrm{~F}\right)\end{array}$ & $\begin{array}{c}L_{k} \\
\left(\times 10^{-3} \mathrm{H}\right)\end{array}$ & $\begin{array}{l}R_{b} \\
(\Omega)\end{array}$ \\
\hline $7.88 \pm 0.14$ & $1.5 \pm 0.2$ & $316 \pm 19$ & $1.98 \pm 0.12$ & $29.1 \pm 0.4$ \\
\hline
\end{tabular}

$=b / k=(6.8 \pm 0.3) \times 10^{-5} \mathrm{~s}$. Note that each variable plays a different role in the behavior of $x_{0} / i_{0}$ : At low frequencies $x_{0} / i_{0} \approx B l / k$; the frequency of resonance is defined primarily by $m / k$; and $b / k$ acts on the sharpness (quality factor) of the resonance peak.

The calculated values of $m / k=a_{2}$ and $b / k=a_{3}$ were then substituted into Eq. (6) to perform the second curve fitting [Fig. 5(b)], resulting in $a_{4}=R=(7.88 \pm 0.14) \Omega, a_{5}=L$ $=(0.15 \pm 0.02) \mathrm{mH}$, and $a_{6}=(B l)^{2} / b=R_{b}=(29.1 \pm 0.4) \Omega$. Note that at low frequencies $Z_{0} \approx R$; at resonance $Z_{0}$ is dominated by the motional impedance and at high frequencies $Z_{0} \approx \omega L$. We checked that the last term of Eq. (6), which has $(B l)^{2} / k=L_{k}$ in the numerator, does not play a significant role in the fitting, so that $L_{k}$ was better calculated by making $L_{k}=a_{3} a_{6}$ and the capacitance $C_{m}=a_{2} / a_{3} a_{6}$. From the quantities reported above we get $B l=a_{3} a_{6} / a_{1}$. All the parameters that characterize the speaker system are summarized in Table I.

It is important to mention that some care is required when using a diode laser as the light source in interferometry. The main advantages of such lasers are their low cost and compactness, which make them well suited for incorporation into undergraduate teaching labs, particularly when several lasers are required at one time. On the negative side, we have found that our diode laser is more unstable than HeNe lasers. Care must also be taken to avoid light returning back to the laser cavity. In addition, small changes in the temperature or the injection current cause a variation in the laser spectrum, and sometimes, two or more longitudinal modes may exist in the laser beam. ${ }^{22}$ As a result, the output voltage of the photodetector, $V_{D}$, may become noisy. In practice, we have not found this to be much of a problem because of the short time required for each measurement point and the ability to change laser parameters such as the injection current and/or the temperature.

The goal is to obtain a well-defined curve on the oscilloscope screen that allows the number of peaks, $n$, to be counted. For that we typically had to wait a few seconds. If desirable, an ultrastable current supply and temperature controller can be mounted to produce a frequency-stable output beam from the laser diode. ${ }^{23,24}$ However, satisfactory results were obtained using the simple commercial laser diode module.

\section{CONCLUSIONS}

We have shown that a speaker system can be characterized by measuring three quantities as functions of the vibrating frequency: the amplitude of the electrical current through the speaker coil terminals, the amplitude of the applied voltage, and the amplitude of the vibration (displacement). Ordinary electrical devices, such as multimeters or oscilloscopes, can be used to measure the current and the applied voltage. The amplitude of the vibration is evaluated by using a very simple laser vibrometer operated by an inexpensive diode laser at the wavelength $\lambda=0.65 \mu \mathrm{m}$. The vibrometer is compact, robust, and also easy to assemble and disassemble. The technique has been experimentally verified on a simple case study, a small low-power loudspeaker. The structural parameters of the speaker were determined, as well as the components of an analog all-electrical network to the speaker system.

\section{ACKNOWLEDGMENTS}

This work was supported by FAPESP_-Fundação de Amparo à Pesquisa do Estado de São Paulo, Brazil (Proc. 97/ 13231-6) and CNPq-Conselho Nacional de Desenvolvimento Científico e Tecnológico, Brazil (PROFIX, Proc. 540294/01-2).

\section{${ }^{a)}$ Electronic mail: afreschi@rc.uncsp.br}

${ }^{1}$ L. E. Drain, The Laser Doppler Technique (Wiley, New York, 1980).

${ }^{2}$ F. Durst, A. Melling, and J. H. Whitelaw, Principles and Practice of LaserDoppler Anemometry (Academic, New York, 1981), 2nd ed.

${ }^{3}$ B. E. Truax, F. C. Demarest, and G. E. Sommargren, "Laser Doppler velocimeter for velocity and length measurements of moving surfaces," Appl. Opt. 23, 67-73 (1984).

${ }^{4}$ A. A. Freschi, A. K. A. Pereira, K. M. Ahmida, J. Frejlich, and J. R. F. Arruda, "Analyzing the total structural intensity in beams using a homodyne laser Doppler vibrometer," Shock Vib. Dig. 7 (5), 299-308 (2000).

${ }^{5}$ T. D. Nichols, D. C. Harrison, and S. S. Alpert, "Simple laboratory demonstration of the Doppler-shift of laser-light," Am. J. Phys. 53 (7), 657660 (1985)

${ }^{6}$ R. H. Belansky and K. H. Wanser, "Laser Doppler velocimetry using a bulk optic Michelson interferometer: A student laboratory experiment," Am. J. Phys. 61 (11), 1014-1019 (1993).

${ }^{7}$ T. J. Belich, R. P. Lahm, R. W. Peterson, and C. D. Whipple, “Optical Doppler measurements," Am. J. Phys. 65 (3), 186-190 (1997).

${ }^{8} \mathrm{~K}$. Dholakia, "An experiment to demonstrate the angular Doppler effect on laser light,” Am. J. Phys. 66 (11), 1007-1010 (1998).

${ }^{9}$ L. E. Kinsler and A. R. Frey, Fundamentals of Acoustics (Wiley, New York, 1962), 2nd ed.

${ }^{10}$ R. P. Feynman, R. B. Leighton, and M. Sands, Lectures on Physics (Addison-Wesley, Reading, MA, 1977), 6th ed.

${ }^{11}$ G. L. Rossi and E. P. Tomasini, "Vibration measurements of loudspeaker diaphragms by a laser scanning vibrometer," Proceedings of the 13th International Modal Analysis Conference, Nashville, 1995, pp. 1205-1209.

${ }^{12}$ G. M. Revel and G. L. Rossi, "Sound power estimation by laser Doppler vibration measurement techniques," Shock Vib. Dig. 5 (5-6), 297-305 (1998).

${ }^{13}$ E. Hecht, Optics (Addison-Wesley-Longman, 1998), 3rd ed.

${ }^{14}$ Part ALA12-3.5G-650, Creative Technology Lasers, 5057 Heather Glen Lane, Concord, CA 94521-3092, 〈www.laser66.com〉. The price per laser is approximately US\$40. We emphasize that regardless of the fact that satisfactory results were obtained using this laser, other types of diode lasers may work as well, and our choice might not be the best one.

${ }^{15}$ Part D32,505, Edmund Scientific (Industrial Optics Division), 101 East Gloucester Pike, Barrington, NJ 08007-1380, U.S., 〈www.edmundoptics.com〉.

${ }^{16}$ Part OPT210P, Burr-Brown, 6730 S. Tucson Blvd., Tucson, AZ 85706, U.S., 〈www.burr-brown.com〉.

${ }^{17}$ CODIT-Silver (Code: 7210), 3M Worldwide, 〈www.3m.com〉. This ink has immersed on it a large amount of spheres, with diameters of some tenths of micrometers. The surface treatment increases the detected signal due to the high reflectivity of this ink and because the incident light is backreflected over a small angle.

${ }^{18}$ Model CFG253 Function Generator, Tektronix, 14200 SW Karl Braun Drive, P.O. Box 500, Beaverton, OR 97077, U.S., 〈www.tek.com〉.

${ }^{19}$ Model TX3 Digital Multimeter, see Ref. 18.

${ }^{20}$ Model TDS420A Digitizing Oscilloscope, see Ref. 18. 
${ }^{21}$ A. Papoulis, Probability, Random Variables, and Stochastic Processes (IEMcGraw-Hill, UK, 1991), 3rd ed.

${ }^{22}$ R. A. Boyd, J. L. Bliss, and K. G. Libbrecht, "Teaching physics with 670-nm diode lasers-experiments with Fabry-Perot cavities," Am. J. Phys. 64 (9), 1109-1116 (1996).
${ }^{23}$ C. C. Bradley, J. Chen, and R. G. Hulet, "Instrumentation for the stable operation of laser diodes," Rev. Sci. Instrum. 61 (8), 2097-2101 (1990).

${ }^{24}$ K. G. Libbrecht and J. L. Hall, "A low-noise high-speed diode laser current controller,” Rev. Sci. Instrum. 64 (8), 2133-2135 (1993).

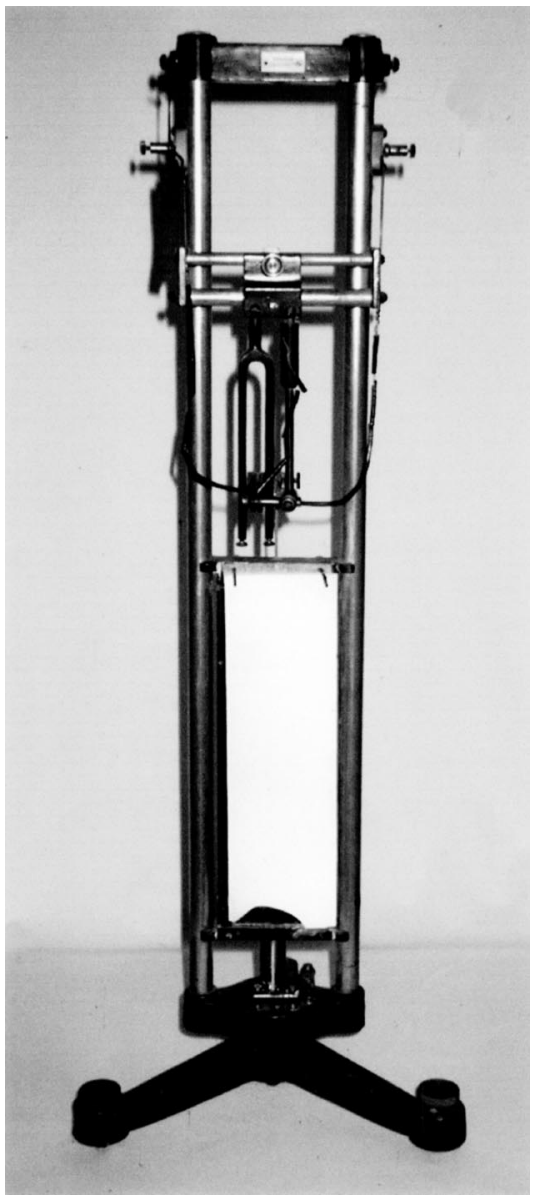

Free Fall Apparatus. The Wm. Gaertner Company of Chicago was usually regarded as a source for good-quality optics apparatus, but in the nineteen twenties it made a line of general laboratory equipment, such as this free-fall apparatus. The glass plate at the bottom was covered with white liquid shoe polish, and allowed to fall past the electrically-maintained tuning fork of known period that can be seen, tines downward, in the upper half of the apparatus. A stylus attached to one tine scratched a trace down the shoe polish layer. From the trace a record of position vs. time could be obtained, from which the value of " $\mathrm{g}$ " could be found. The apparatus is in the Greenslade Collection. (Photograph and notes by Thomas B. Greenslade, Jr., Kenyon College) 